\title{
Phylogeny of the bodonid flagellates (Kinetoplastida) based on small-subunit rRNA gene sequences
}

\author{
David Doležel, ${ }^{1,2} †$ Milan Jirků, ${ }^{1,2}$ Dmitri A. Maslov ${ }^{3}$ and Julius Lukešr,2
}

Author for correspondence: Dmitri A. Maslov. Tel: +1909 787 6485. Fax: + 19097874286.

e-mail:maslov@ucracl.ucr.edu

1 Institute of Parasitology, Czech Academy of Sciences, České Budějovice, Czech Republic

2 Faculty of Biology, University of South Bohemia, Ceské Budějovice, Czech Republic

3 Department of Biology, University of California, Riverside, CA 92521, USA

\begin{abstract}
The phylogeny of kinetoplastid flagellates was investigated by determining the sequences of the small-subunit (18S) rRNA from Bodo designis, Bodo saltans K, Bodo saltans P, Bodo sorokini, Bodo sp. (cf. uncinatus), Cruzella marina, Cryptobia helicis, Dimastigella mimosa and Parabodo nitrophilus and analysing these data together with several previously obtained sequences. The root of the kinetoplastid tree was tentatively determined to be attached to the branch of B. designis and/or Cruzella marina. Within this topology, the suborder Trypanosomatina appears as a late-emerging monophyletic group, while the suborder Bodonina is paraphyletic. Within the bodonid subtree, the branches of parasitic organisms were intermingled with free-living ones, implying multiple transitions to parasitism. The tree indicates that the genera Cryptobia and Bodo are artificial taxa. In addition, the separation of the fish cryptobias and Trypanoplasma borreli as different genera was not supported.
\end{abstract}

Keywords: Kinetoplastida, Bodonina, Bodo, Cryptobia, phylogeny

\section{INTRODUCTION}

Studies of protozoa from the order Kinetoplastida (phylum Euglenozoa) has yielded a large number of unexpected findings, probably more than for any other comparable group of protists (Vickerman, 1994; Donelson et al., 1999). The order has been defined by the presence of a unique organelle, the kinetoplast, which represents a DNA-containing compartment of the single mitochondrion of a cell.

These studies have revealed organization of the mitochondrial (kinetoplast) DNA in the form of giant networks, an unusual uridylate insertion/deletion type of RNA editing, intriguingly small kinetoplast rRNAs,

\footnotetext{
†Present address: Institute of Entomology, Czech Academy of Sciences, České Budějovice, Czech Republic.

Abbreviations: kDNA, kinetoplastid DNA; LSU large-subunit; SSU, smallsubunit; T-PTP, topology-dependent cladistic permutation tail probability. The GenBank accession numbers of the determined SSU rRNA sequences are: AF209856 (B. designis), AF208878 (C. marina), AF208880 (C. helicis), AF208882 (D. mimosa), AF208884 [Bodo sp. (cf. uncinatus)], AF208886 ( $P$. nitrophilus), AF208887 (B. saltans P), AF208888 (B. sorokini), AF208889 (B. saltans K); and of the determined partial LSU rRNA sequences are: AF208879 (C. marina), AF208881 (C. helicis), AF208883 (D. mimosa), AF208885 (P. nitrophilus), AF208890 (B. saltans K).
}

importation of all mitochondrial tRNAs from the cytosol, trans-splicing of cytosolic mRNAs, trypanosome surface antigen variation and a novel nucleotide in DNA referred to as base $\mathrm{J}$ (reviewed by Donelson et al., 1999) with this list likely to be continued in the future. Due to the early separation of Euglenozoa (Sogin et al., 1986), some of these features could have been directly inherited from the early eukaryotes, while others can represent more recent adaptations, having evolved, for example, as an adaptation to facultative anaerobiosis (Cavalier-Smith, 1997) or even at later stages, as adaptations to parasitism.

Based on morphology, Kinetoplastida is traditionally divided into two suborders - Bodonina and Trypanosomatina (Vickerman, 1976, 1978; Lom, 1979; Kivic \& Walne, 1984). Members of Trypanosomatina are characterized as obligatory parasitic species with a single flagellum and a relatively small kinetoplast, while species of Bodonina include free-living, commensal and ecto- and endoparasitic species with two flagella and a larger kinetoplast.

Because of their importance to human and animal health, trypanosomatids have been studied more intensively than bodonids. Investigation of trypanosomatid evolution at the molecular level included the 
analysis of rRNA gene sequences from more than fifty trypanosome species (reviewed by Stevens et al., 1999). Early work addressed general aspects of the evolution of parasitism (Fernandes et al., 1993; Maslov \& Simpson, 1995; Maslov et al., 1996) and investigated evolution of RNA editing (Landweber \& Gilbert, 1994; Maslov et al., 1994). Special attention has been paid to the evolution of the genus Trypanosoma with its more advanced characteristics and diverse parasitic adaptations (Lukeš et al., 1997; Haag et al., 1998; Stevens et al., 1999). These works also shed light on the evolution of bacterial endosymbiosis in this group (Du et al., 1994). Several studies refined the trypanosomatid taxonomy by showing that some genera (such as Crithidia and Herpetomonas) are polyphyletic and represent artificial taxa (Hollar et al., 1998), while others (Trypanosoma and Phytomonas) are monophyletic and represent natural taxa (Hollar \& Maslov, 1997; Lukeš et al., 1997).

Bodonids, on the other hand, have attracted substantially less attention. So far, sequences useful for phylogenetic analyses are available only for the fish parasites Trypanoplasma borreli (Maslov et al., 1994) and Cryptobia spp. (Wright et al., 1999), and the freeliving species Bodo caudatus (Fernandes et al., 1993), Dimastigella trypaniformis (Berchtold et al., 1994) and 'Rhynchobodo' sp. (Lukeš et al., 1997). By putting into phylogenetic context the data obtained by the analysis of RNA editing (Maslov et al., 1994; Lukeš et al., 1994; Blom et al., 1998, 2000), kDNA structure (Yasuhira \& Simpson, 1996; Lukeš et al., 1998), and glycosomes (Wiemer et al., 1995) a better understanding of these processes and structures can be achieved. The aim of this work is to determine phylogenetic relationships within the suborder Bodonina, and to provide a framework for molecular and biochemical studies on bodonids.

\section{METHODS}

Strain origin and cultivation conditions. Bodo saltans strain St Petersburg (P), Dimastigella mimosa and Parabodo nitrophilus were isolated from the tanks of a sewage plant in Borok, Yaroslavl, Russia (Frolov et al., 1997; Mylnikov, 1986). Bodo designis was isolated from the soil in St Petersburg, Russia, in 1992. All the aforementioned strains were provided by S. Karpov (Institute of Cytology, St Petersburg, Russia) and A. Frolov (Zoological Institute, St Petersburg, Russia). Bodo sp. (cf. uncinatus) represents the strain ATCC 30904 (Daggett \& Nerad, 1982). It was originally isolated from a freshwater pool and provided to us by J. Kulda (Faculty of Sciences, Charles University, Prague). D. mimosa and Bodo sp. (cf. uncinatus) were cultivated in the ATCC medium 802 with the feeder bacteria Alcaligenes xylosoxidans denitrificans and Aerobacter aerogenes at 16 and $20^{\circ} \mathrm{C}$, respectively. The origin and cultivation of the Bodo saltans strain Konstanz (K) have been described previously (Blom et al., 1998). 'Rhynchobodo' sp. (ATCC strain 50359) and Cruzella marina (ATCC strain 50326) were obtained from the American Type Culture Collection. The ultrastructural examination of 'Rhynchobodo' sp. demonstrated its similarity with other species of
Bodo, especially with B. designis (A. Mylnikov, personal communication). Inclusion of $C$. marina in kinetoplastids is supported by analysis of the kinetoplast DNA (A. Žiková, M. Jirků \& J. Lukeš, unpublished). The strain Bodo sorokini ATCC 50641 was isolated from marine sediments of the White Sea, Tshupa Bay, Russia, in 1986, and obtained from A. Mylnikov (Institute of Biology of Inland Waters, Borok, Russia). B. sorokini (Zhukov, 1975) is morphologically and ultrastructurally close to $B$. caudatus and $P$. nitrophilus (A. Mylnikov, personal communication). C. marina, $B$. designis and $B$. sorokini were cultivated with the feeder bacteria $A$. x. denitrificans in the ATCC medium 1525 at 16 or $22^{\circ} \mathrm{C}$ (B. sorokini). The origin of Cryptobia helicis has been described elsewhere (Lukeš et al., 1998).

DNA extraction, amplification and sequencing. Isolation of total cell DNA, PCR amplification of the small-subunit (SSU) rRNA genes and partial large-subunit (LSU) rRNA genes and sequencing with a set of conserved oligomer primers were performed as described previously (Maslov et al., 1996, 1999; Lukeš et al., 1997).

Phylogenetic analysis. Additional 18S rRNA sequences used in this work were retrieved from GenBank and include: the trypanosomatids - Crithidia fasciculata (X03450), Herpetomonas muscarum (L18872), Phytomonas serpens strain 1G (AF016323), Herpetomonas roitmani (AF038023) and Trypanosoma cruzi (M31432); the bodonids - Dimastigella trypaniformis (strain Ulm - X76494, strain Glasgow X76495), 'Rhynchobodo' sp. (U67183), Trypanoplasma borreli (L14840), Cryptobia bullocki (AF080224), Cryptobia catostomi (AF080226), Cryptobia salmositica (AF080225), Bodo caudatus (X53910); and the diplonemids - Diplonema papillatum (AF119811) and Diplonema sp. (AF119812).

Alignments were generated manually using an interactive multiple alignment editor SeqEdit, version 3.1 (Olsen, 1990). The alignments are available on request from D. A. M. or can be retrieved from the following URL: http://www. lifesci.ucla.edu/RNA/trypanosome/alignments.html/. Maximum-likelihood and parsimony analyses were performed using PAUP* 4.0 beta version (Swofford, 1998) with the outgroup represented as a monophyletic sister clade to the ingroup. A heuristic search was performed for likelihood trees, and branch-and-bound search for parsimony trees. The corresponding bootstrap analyses included 100 or 500 replicates, respectively. For the parsimony analysis, an additional evaluation of monophyletic groups was performed using T-PTP (topology-dependent cladistic permutation tail probability). This test included 500 permutations (Faith, 1991). The null hypothesis (non-monophyly) can be rejected at $P \leqslant 0.05$. Statistical evaluation of the trees inferred under different topological constraints was performed using the Kishino-Hasegawa test (Kishino \& Hasegawa, 1989). The null hypothesis (absence of significant differences between the trees in question) is also rejected at $P \leqslant 0.05$. Both statistical tests are included in the PAUP package.

\section{RESULTS}

\section{Alignments}

Two alignments of the SSU rRNA sequences were constructed and used for analysis of the kinetoplastid phylogeny. The first alignment contained 1283 characters for the 22 kinetoplastid ingroup and two diplo- 


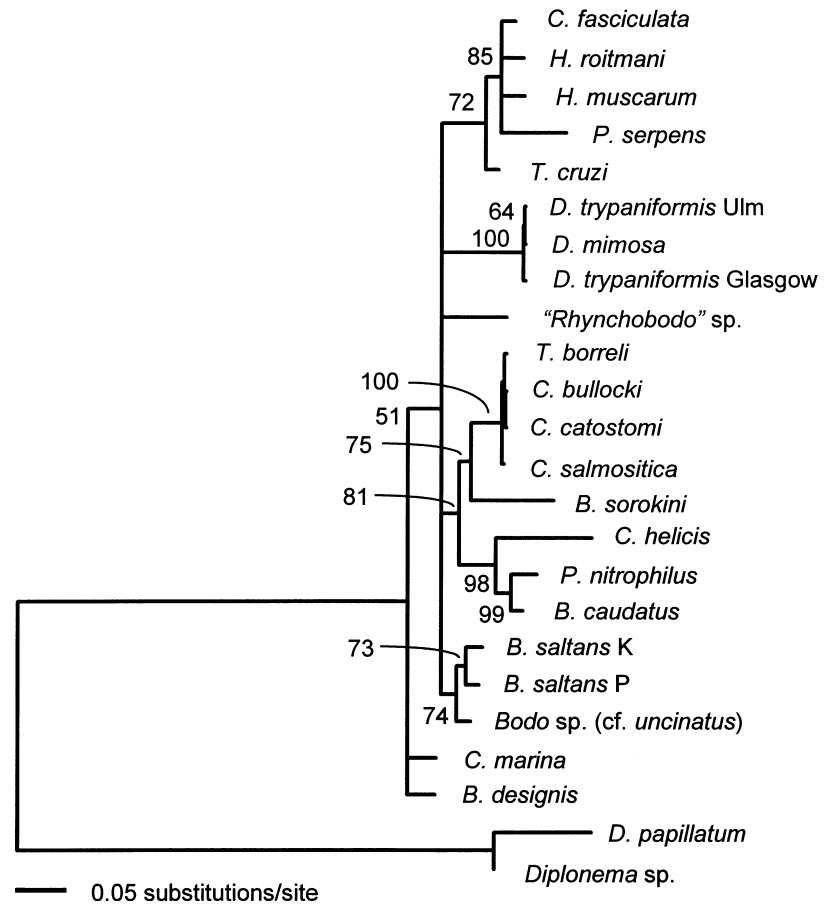

Fig. 1. Majority consensus $(50 \%)$ maximum-likelihood tree of Kinetoplastida constructed using the SSU rRNA alignment with 1283 characters and containing two diplonemid outgroup species. The bootstrap values determined for 100 replicates are given at each node. The distance scale is given under the tree. Other parameters were as described in Table 1 for unconstrained search with the full taxon set.

nemid outgroup taxa. The close relationship of the orders Kinetoplastida and Diplonemida within Euglenozoa is well supported by morphology and ultrastructure (Kivic \& Walne, 1984; Simpson, 1997) and corroborated by the SSU rRNA and cytochrome oxidase I (COI) protein phylogenetic analysis (Maslov et al., 1999). The ingroup contained nine new and eight known bodonid sequences. Although more than 50 trypanosomatid sequences are currently available, for practical reasons we have included only five sequences which were representative of the five major monophyletic clades of trypanosomatids: T. cruzi for trypanosomes; $H$. roitmani for the endosymbiont-bearing clade; $P$. serpens for phytomonads, H. muscarum for the endosymbiont-free herpetomonads and C. fasciculata for the clade of crithidias, leishmanias and related groups (Hollar et al., 1998). The purpose of this alignment was to find the root of the kinetoplastid tree. The second alignment contained only 22 kinetoplastid taxa. With the absence of the outgroup it was possible to align a larger number of characters (1693), thus producing an alignment which was more informative for investigating relationships within the ingroup.

Our preliminary analyses also included three species of Euglenida, as we did in the previous work (Maslov et al., 1999). However, we found that the trees obtained (data not shown) were less robust with lower bootstrap values and a more frequent occurrence of polytomies, than trees rooted with diplonemids. At the same time the root of the maximum-likelihood tree and topology of the ingroup were identical as with the use of diplonemids only. Since the euglenoids formed the longest branches in the tree, it seemed likely that a decrease in resolution was due to a larger proportion of sites with mutational saturation in the kinetoplastid sequences versus the outgroup. We, therefore, omitted the euglenids from further analyses.

\section{The root of the kinetoplastid tree}

The maximum-likelihood bootstrap consensus tree for 22 ingroup and two outgroup taxa is shown in Fig. 1. In this case, the position of the root can be only marginally resolved with the ribosomal dataset. The basal trichotomy includes the branches of $C$. marina and $B$. designis, as well as the clade containing all the remaining kinetoplastids. However, this topology is seen only in $51 \%$ of bootstrap replicates indicating a low level of statistical support. In addition, the position of the root was sensitive to the set of assumptions for the likelihood analysis: the root was obtained in this position only under the assumption of variable rates following $\gamma$-distribution with the shape parameter estimated via likelihood. Two other parameters, the proportion of invariable sites and the transition/ transversion ratio, were also estimated by likelihood. Any simplification of assumptions resulted in a tree with a basal polytomy (data not shown) which included not only the branches of $C$. marina and B. designis, but also several individual branches and clades of the remaining kinetoplastids. Bootstrapping with maximum-parsimony produced an unresolved tree. These results suggest that the amount of phylogenetic information which determines a correct position of the root is very low, so that only a proven power of maximum-likelihood, most of all its reduced sensitivity to misleading multiple nucleotides changes, unequal rates and sampling variance (Swofford et al., 1996), could be used to discern this phylogenetic signal.

The best maximum-likelihood tree (data not shown) revealed the same trichotomy, as seen in the consensus tree (B. designis, C. marina and the clade of remaining species). This result, and the results of the bootstrap analysis, indicated that either B. designis or C. marina, or the clade uniting both of them represents the earliest offshoot from the kinetoplastid tree. These topologies make the bodonids a paraphyletic assembly and the trypanosomatids a late diverging monophyletic clade. However, given the uncertainty regarding the root position, it was necessary to investigate alternative $a$ priori hypotheses. If the root were attached at the internal branch between bodonids and trypanosomatids, this would make both groups monophyletic, and if the root were attached at the trypanosomatid clade, this group would be paraphyletic, while bodonids would constitute a monophyletic group. Testing these possibilities was done by imposing the corresponding 
Table 1. Parameters of the phylogenetic trees produced with maximum likelihood and parsimony

\begin{tabular}{|c|c|c|c|c|c|}
\hline \multirow[t]{3}{*}{ Topological constraint } & \multicolumn{2}{|c|}{ Maximum likelihood } & \multicolumn{3}{|c|}{ Parsimony } \\
\hline & \multirow{2}{*}{$\begin{array}{l}\text { In likelihood } \\
\text { (no. trees) }\end{array}$} & \multirow{2}{*}{$\begin{array}{l}P \text {-value in } \\
\mathrm{K}-\mathrm{H} \text { test }\end{array}$} & \multirow{2}{*}{$\begin{array}{l}\text { No. steps } \\
\text { (no. trees) }\end{array}$} & \multicolumn{2}{|c|}{$P$-value in } \\
\hline & & & & T-PTP & $\mathrm{K}-\mathrm{H}$ test* \\
\hline \multicolumn{6}{|l|}{ Full taxon set: } \\
\hline No constraints & $-5822 \cdot 20292(1) \dagger$ & - & $829(2)$ & - & - \\
\hline \multicolumn{6}{|l|}{ Constraints } \\
\hline$(\mathrm{O},(\mathrm{B},(\mathrm{T})))$ & $-5822 \cdot 20292(2) \ddagger$ & - & $829(1)$ & - & - \\
\hline$(\mathrm{O},(\mathrm{T},(\mathrm{B})))$ & $-5822 \cdot 51861(1)$ & $0 \cdot 9172$ & $833(6)$ & $0 \cdot 190$ & $0 \cdot 4654-0.5373$ \\
\hline$(\mathrm{O},((\mathrm{B}),(\mathrm{T})))$ & $-5823 \cdot 17311(1)$ & $0 \cdot 7214$ & $829(1)$ & - & - \\
\hline \multicolumn{6}{|l|}{ Ingroup set: } \\
\hline No constraints & $-8085 \cdot 45042(1) \S$ & - & $1151(2)$ & - & - \\
\hline \multicolumn{6}{|l|}{ Constraints for the monophyly of: } \\
\hline $\begin{array}{l}\text { (1) Trypanosomatids with } B \text {. } \\
\text { saltans/uncinatus }\end{array}$ & $-8090 \cdot 40946(1)$ & $0 \cdot 3852$ & $1157(2)$ & $0 \cdot 044$ & $0 \cdot 1088,0 \cdot 1574$ \\
\hline $\begin{array}{l}\text { (2) Trypanosomatids with the entire clade } \\
\text { of Trypanoplasma/Parabodo }\end{array}$ & $-8090 \cdot 43298(1)$ & $0 \cdot 3796$ & $1159(4)$ & $0 \cdot 052$ & $\begin{array}{l}0 \cdot 1306,0 \cdot 1167 \\
0 \cdot 0325,0 \cdot 0209\end{array}$ \\
\hline (3) All Cryptobia with T. borreli & $-8107 \cdot 19467(1)$ & $0 \cdot 0298$ & $1162(2)$ & $0 \cdot 230$ & $0 \cdot 1086,0 \cdot 1011$ \\
\hline $\begin{array}{l}\text { (4) Trypanosomatids with } T \text {. borreli and } \\
\text { fish Cryptobia }\end{array}$ & $-8144 \cdot 63156(1)$ & $0 \cdot 0001$ & $1176(1)$ & 0.616 & $0 \cdot 0011$ \\
\hline (5) All Bodo & $-8385 \cdot 32948(1)$ & $<0.0001$ & $1256(4)$ & $1 \cdot 000$ & $<0 \cdot 0001$ \\
\hline
\end{tabular}

* Multiple values appear in comparisons involving several suboptimal (constrained) trees.

$\uparrow$ The tree parameters were estimated via likelihood and included: transition/transversion ratio (1.602427), value of proportion of invariable sites $(0 \cdot 375400)$, value of $\gamma$-shaped parameter $(0.524818)$. The same values of these parameters were set in the search of the trees with topological constraints.

$\$$ One of the trees is rooted at B. designis, and the other has the trichotomy which also includes C. marina and the monophyletic clade of all remaining kinetoplastids. In most other cases, the differences were restricted to the internal branching order of trypanosomatid clade or the clade of Trypanoplasma/fish Cryptobia.

$\S$ The estimated tree parameters were: transition/transversion ratio (1.621341), value of proportion of invariable sites $(0 \cdot 456734)$, value of $\gamma$-shaped parameter $(0 \cdot 605804)$.

topological constraints on the tree, and the results are given in Table 1. The best unconstrained tree and the trees with a constraint for the paraphyly of Bodonina show the same highest value of ln likelihood. This value was slightly lower under the constraint for the paraphyly of trypanosomatids, and it was even more so when both groups were forced to be monophyletic. According to the Kishino-Hasegawa test, these differences were not significant. The parsimony analysis showed that the paraphyly of Bodonina and the monophyly of both groups represented two most parsimonious topologies, while the paraphyly of trypanosomatids did not result in a significantly different score (Table 1). The T-PTP test, however, did not support the monophyly of bodonids which is implied by the latter topology. So, we can tentatively conclude that the tree topology with the root attached to bodonids, and within bodonids to the B. designis/C. marina branch, is most supported. However, the differences between alternative topologies were subtle, indicating that the problem of the earliest kinetoplastid branches was only marginally resolved by this analysis. Finding a closer outgroup would be needed for a more reliable rooting of the rRNA tree.

\section{Relationships within the Kinetoplastida}

A well-resolved tree topology was obtained with maximum-likelihood (Fig. 2), and to a lesser extent with maximum-parsimony, when the outgroup taxa were excluded from the alignment, thus allowing for a substantial increase of the number of alignable characters. This fact also indicates that the polytomies obtained with the previous dataset reflect a shortage of informative characters in that alignment rather than a real burst in the kinetoplastid radiation.

The ingroup topology consisted of several highly supported monophyletic clades which included the clade of trypanosomatids, the clade of $T$. borreli, fish Cryptobia, B. sorokini and P. nitrophilius together with the sister clade of $B$. saltans and Bodo sp. (cf. uncinatus), and the clade of B. designis and C. marina. Only the positions of the Dimastigella species and 'Rhynchobodo' sp. remained incompletely resolved. These organisms grouped together in $47 \%$ of replicates, and in $50 \%$ of cases Dimastigella spp. were monophyletic with $B$. designis and $C$. marina.

The most remarkable feature of the tree obtained was 

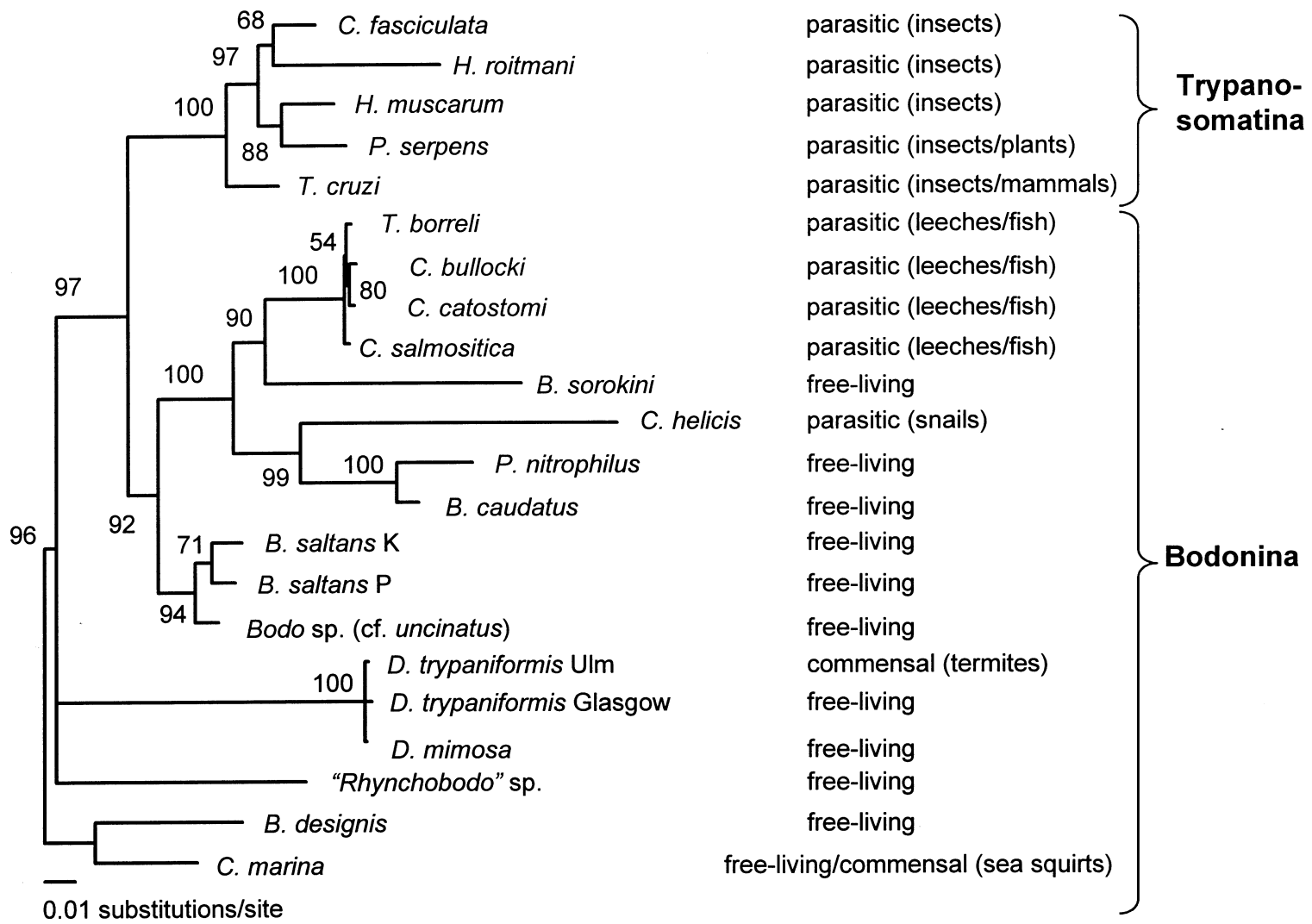

Fig. 2. Majority consensus maximum-likelihood SSU rRNA tree of Kinetoplastida constructed using the alignment with 1693 characters and only the ingroup species. Indication of the lifestyle and the hosts is shown on the right. The tree parameters were as described in Table 1 for unconstrained search with the ingroup set.

that the parasitic organisms did not form a separate cluster. The parasitic and the commensal bodonids were found interspersed among the free-living species within the large Trypanoplasma-Cryptobia-BodoParabodo clade (Fig. 1). The topological constraint that enforced the monophyly of $C$. helicis from snails and the compact Trypanoplasma-fish Cryptobia group (Table 1, constraint 3) resulted in a tree which was significantly less likely using maximum-likelihood. Although the corresponding difference (11 steps) in parsimony was not significant, the monophyly still did not withstand the T-PTP test. Nor was there any support for a closer grouping of these fish parasites with the trypanosomatids (constraint 4). Alternative topologies, which could not be excluded a priori, were the monophyly of trypanosomatids with the clade of free-living $B$. saltans/Bodo sp. (cf. uncinatus) (constraint 1) and, to a lesser extent, the monophyly of trypanosomatids with the mixed clade of Trypanoplasma-Cryptobia-Bodo-Parabodo (constraint 2). From a taxonomic perspective, these results demonstrated the polyphyly of the genus Cryptobia (constraint 3).

Even more striking was the polyphyly of the genus Bodo, the members of which were found within four different monophyletic clades, all strongly supported; the trees constrained for the monophyly of Bodo (constraint 5) were significantly inferior to the unconstrained trees. At the same time, the close relatedness of the geographically distant isolates of Dimastigella spp. supported their taxonomic assignment into the same genus, although there was no consistency on the level of species. Similarly, the B. saltans strains originating from Germany and Russia were monophyletic, although the intraspecific sequence differences were larger than the interspecific differences among the fish cryptobias. An observed close relatedness of the latter group with $T$. borreli could be expected, since based on their morphology, these organisms are sometimes considered members of the same genus (see also Discussion). Results of a preliminary analysis with the limited set of the LSU sequences (data not shown) confirmed the phylogenetic relationships of $C$. marina, $C$. helicis, $D$. mimosa, $P$. nitrophilus and $B$. saltans inferred from the SSU sequences.

\section{DISCUSSION}

As members of all major aquatic and terrestrial ecosystems (Foissner, 1991), bodonids represent an ecologically and economically important group of organisms. They are also crucial components of sewage cleaning units and the causative agents of fish diseases 
in aquacultures (Woo, 1994). In an attempt to better understand the phylogenetic relationships within this group, we have investigated a variety of free-living, commensal, and parasitic species of bodonids from geographically distant localities. Our results show that bodonids are most likely paraphyletic, while trypanosomatids, in agreement with the current morphologybased taxonomy, represent a late-emerging monophyletic group. The tree is rooted at the free-living organisms $B$. designis and C. marina and also contains a number of free-living bodonids at its base.

The problem of mutational saturation, which has hampered many rRNA-based phylogenetic analyses (Philippe \& Adoutte, 1998), seems to be present in our analysis as well, and may be responsible for the difficulties in determining a position of the root. The topology of the tree (Fig. 1) showing multifurcation of lineages with similar lengths indicates mutational saturation in the ingroup with regard to the outgroup. It is remarkable that by removing the outgroup, thereby reducing the number of sites with multiple mutations, a better resolved tree (Fig. 2) can be obtained. Additional problems associated with reconstructions of trypanosomatid phylogeny using rRNA data are caused by 'long branch attraction' which occurs when substitution rates in some lineages far exceed those in others (Felsenstein, 1978; Maslov et al., 1996; Lukeš et al., 1997). It seems that this artefact is not directly responsible for the observed root of the maximum-likelihood trees, as the branches of $C$. marina and $B$. designis are relatively short. However, the long branch attraction may be responsible for an unstable position of the Dimastigella species and 'Rhynchobodo' sp. (see Results) which group together in $47 \%$ of bootstrap replicates and the branches of which are among the longest in the tree (Fig. 2). In addition, this artefact is likely to produce the observed root in the corresponding parsimony trees (Table 1) in which it is attached to the branch of 'Rhynchobodo' sp. (not shown).

Analysis of the topologically constrained trees shows that the root attached at the bodonids is only slightly preferred to alternative models that include the root attached between the clades of bodonids and trypanosomatids or even at trypanosomatids. Considering the last two models, a trypanosomatid root is less plausible, even though it has a higher support with likelihood (Table 1), since this scenario would contradict abundant morphological, ultrastructural, molecular and ecological data indicating the derived nature of trypanosomatids. On the other hand, the best tree topology confirms earlier hypotheses that the ancestral kinetoplastids were free-living bodonid-like organisms (Vickerman, 1994).

It is clear that a resolution of this problem will require finding an outgroup more closely related to kinetoplastids or analysing another dataset. It should be noted that the analysis of several mitochondrial genes (Lukeš et al., 1994; Blom et al., 1998) also supported the bodonid root. Unfortunately, the limited number of available protein sequences is insufficient for a more detailed analysis.

However, the unrooted topology of the kinetoplastid SSU rRNA tree is very robust and, assuming that it reflects a true phylogenetic history of the group, allows several important conclusions to be drawn concerning the origin and evolution of parasitism in Kinetoplastida, even in the absence of a well-defined root. Parasitic bodonids, such as $T$. borreli and three species of fish cryptobias on one hand, and $C$. helicis on another, belong to the separate monophyletic clades. Trypanosomatids, all of which are parasitic, also form a separate clade within Kinetoplastida. This topology is well supported by the data and it strongly suggests that several and apparently independent acquisitions of the parasitic lifestyle occurred during the evolution of kinetoplastid flagellates. Moreover, it seems that this process still continues and the different steps of it can be observed in the existing clades. The examples of $C$. marina and D. trypaniformis, for which both endocommensal and free-living strains have been described (De Faria et al., 1922; Nerad, 1993; Vickerman, 1978; Breunig et al., 1993), may reflect the very early stages of transition towards endoparasitism. A more advanced step in this direction is illustrated by the obligatory endocommensal $C$. helicis which is likely to have evolved from a bodonid with a free-living lifestyle still preserved in the related species $B$. caudatus, $P$. nitrophilus and $B$. sorokini. Another transition to parasitism has independently occurred in the lineage leading towards the $T$. borreli-fish Cryptobia group. In this case, the evolution reached the stage of transition from intestinal parasites to haemoparasites, as this compact group contains both types of organisms. All these events seem to correspond to the early hypothesis of Minchin, who postulated that the haemoparasites of vertebrates evolved from their endoparasites, which in turn were derived from the free-living species (Minchin, 1908). The fact that none of the groups illustrates the entire evolutionary process should not be surprising. First, the intermediate stages might have become extinct, and second, the present survey of extant kinetoplastids is far from being complete.

The tree supports an independent origin of parasitism in trypanosomatids. This group of obligatory parasites is well separated in the phylogenetic tree from any group of parasitic bodonids. The data almost exclude the origin of trypanosomatids from Cryptobia-like bodonids, a scenario derived from the ultrastructural analysis (Kivic \& Walne, 1984). The only bodonid clade which may be more related to trypanosomatids than to other bodonids is the clade of free-living $B$. saltans /Bodo sp. (see below). If the evolution in this case had also followed Minchin's scenario, then organisms representing the intermediate stages, such as endoparasitic trypanosomatids of vertebrates, either remain unknown or have become extinct. Alternatively, the evolution of trypanosomatids might have followed the route outlined by Léger (1904), according 
to which the original parasites were infecting the gut of insects with the subsequent acquisition of haemoparasitism in vertebrates.

The most obvious taxonomic implication of this work is the lack of support for the division of bodonids into two families Bodonidae and Cryptobiidae (Vickerman, 1976, 1978), and the polyphyly of the genus Bodo. The ubiquitous and ecologically important members of this genus appear dispersed throughout the tree as four separate branches. This is quite unexpected, since based on their morphology, the Bodo species are rather uniform, although this may reflect the absence of any peculiar traits rather than the presence of specific unifying characters. However, any result which involves a single isolate from a species or a group of species should be considered as preliminary, since there is always a possibility of a strain misidentification. From this point of view, only the groups of B. saltans, fish cryptobias and Dimastigella spp., each of which represented by two or three isolates, do not cause any concern. From the previous analysis of mitochondrial cox2 gene sequences, B. saltans and Bodo sp. (cf. uncinatus) were inferred to be more closely related to trypanosomatids than to $C$. helicis or T. borreli (Blom et al., 1998). This conclusion was indirectly strengthened for $B$. saltans by the gene order, RNA editing patterns, and structure of the kDNA minicircles which resemble those of trypanosomatids (Blom et al., 1998, 2000). As shown by the results of T-PTP and Kishino-Hasegawa tests (Table 1), the monophyly of B. saltans and Bodo sp. (cf. uncinatus) clade with trypanosomatids cannot be excluded, so the question remains open.

$D$. trypaniformis and D. mimosa differ in several morphological features ranging from the specific position of the cytostome as seen in light microscope to ultrastructural differences of the cytoskeleton and the number of mitochondria (Vickerman, 1978; Frolov et al., 1997). Interestingly, in spite of such morphological variation, $D$. mimosa branch is invariably located between the Glasgow and Ulm strains of $D$. trypaniformis in the best maximum-likelihood and parsimony trees (data not shown). The Ulm strain was isolated from the hindgut of a termite while all other Dimastigella isolates are free-living. However, although differences in growth conditions and in ultrastructure of nucleus between the two strains have been described (Breunig et al., 1993), the authors did not consider these features significant enough for description of a new species. This view was upheld even when $1.9 \%$ differences were found in the 18S rRNAs of both strains (Berchtold et al., 1994). Our results indicate that the taxonomic status of the Ulm strain of $D$. trypaniformis needs to be reconsidered.

In agreement with the previous analysis (Wright et al., 1999), T. borreli and three haematozoic Cryptobia spp. formed a strongly supported monophyletic group, while $C$. helicis, a type species of the genus Cryptobia, constituted a separate branch. The differences in morphology and life cycles between the genera Cryp- tobia and Trypanoplasma have been embraced as sufficient by several authors (e.g. Lom, 1976; Becker, 1977; Brugerolle et al., 1979), while deemed insufficient by others (e.g. Bower \& Margolis, 1983; Woo, 1994). Recently described differences in the kDNA structure between T. borreli (Maslov \& Simpson, 1994; Yasuhira \& Simpson, 1996) and C. helicis (Lukeš et al., 1998), along with the 18S and 28S rRNA data are considered by us as substantial. We therefore suggest that the subgeneric status of Trypanoplasma within the genus Cryptobia, as proposed by Woo (1994), is inadequate, and that the genus Trypanoplasma created early in the last century for biflagellated haematozoic species with a two-host life cycle (Laveran \& Mesnil, 1901) should be considered valid.

\section{ACKNOWLEDGEMENTS}

We thank Drs A. Frolov, S. Karpov, A. Mylnikov, J. Kulda, K. Simek and T. Nerad, who provided strains of bodonids used in this study, and Dr M. Peris for comments on the manuscript. The work was supported by the Grant Agency of the Czech Academy of Sciences no. A6022903 to J. L. and also in part by a Burroughs Wellcome New Investigator Award in Molecular Parasitology and the grant AI40634 from the National Institutes of Health to D.A.M.

\section{REFERENCES}

Becker, C. D. (1977). Flagellate parasites of fish. In Parasitic Protozoa, vol. 1, pp. 375-416. Edited by J. P. Kreier. New York: Academic Press.

Berchtold, M., Philippe, H., Breunig, A., Brugerolle, G. \& König, H. (1994). The phylogenetic position of Dimastigella trypaniformis within the parasitic kinetoplastids, Parasitol Res 80, 672-679.

Blom, D., de Haan, A., van den Berg, M., Sloof, P., Jirků, M., Lukeš, J. \& Benne, R. (1998). RNA editing in the free-living bodonid Bodo saltans, Nucleic Acids Res 26, 1205-1213.

Blom, D., de Haan, A., van den Burg, J., van den Berg, M., Sloof, P., Jirků, M., Lukeš, J. \& Benne, R. (2000). Mitochondrial minicircles in the free-living bodonid Bodo saltans contain two gRNA gene cassettes and are not found in large networks, $R N A$ 6, 1-15.

Bower, S. M. \& Margolis, L. (1983). Direct transmission of the haemoflagellate Cryptobia salmositica among Pacific salmon (Oncorhynchus spp.), Can J Zool 61, 1242-1250.

Breunig, A., Brugerolle, G., Vickerman, K., Hertel, H. \& König, H. (1993). Isolation and ultrastructural features of a new strain of Dimastigella trypaniformis Sandon 1928 (Bodonina, Kinetoplastida) and comparison with a previously isolated strain, Eur J Protistol 29, 416-424.

Brugerolle, G., Lom, J., Nohynková, E. \& Joyon, L. (1979). Comparaison et évolution des structures cellulaires chez plusieurs espèces de Bodonidés et Cryptobiidés appartenant aux genres Bodo, Cryptobia et Trypanoplasma (Kinetoplastida, Mastigophora), Protistologica 15, 197-221.

Cavalier-Smith, T. (1997). Cell and genome coevolution: facultative anaerobiosis, glycosomes and kinetoplastan RNA editing, Trends Genet 13, 6-9.

Daggett, P.-M. \& Nerad, T. A. (1982). Axenic cultivation of Bodo edax and Bodo uncinatus and some observations on feeding rate in monoxenic culture, J Protozool 29, 290. 
De Faria, J. G., Da Cunha, A. M. \& Pinto, C. (1922). Estudos sobre protozoairos do mar, Mem Inst Osw Cruz 15, 186-208.

Donelson, J. E., Gardner, M. J. \& El-Sayed, M. N. (1999). More surprises from Kinetoplastida, Proc Natl Acad Sci USA 96, 2579-2581.

Du, Y., Maslov, D. A. \& Chang, K. P. (1994). Monophyletic origin of $\beta$-division proteobacterial endosymbionts and their coevolution with insect trypanosomatid protozoa Blastocrithidia culicis and Crithidia spp, Proc Natl Acad Sci USA 91, 8437-8441.

Faith, D. P. (1991). Cladistic permutation tests for monophyly and non-monophyly, Syst Zool 40, 366-375.

Felsenstein, J. (1978). Cases in which parsimony or compatibility methods will be positively misleading, Syst Zool 27, 401-410.

Fernandes, A. P., Nelson, K. \& Beverley, S. M. (1993). Evolution of nuclear ribosomal RNAs in kinetoplastid protozoa: perspectives on the age and origins of parasitism, Proc Natl Acad Sci USA 90, 11608-11612.

Foissner, W. (1991). Diversity and ecology of soil flagellates. In The Biology of Free-living Heterotrophic Flagellates, pp. 93-112. Edited by D. J. Patterson \& J. Larsen. Oxford: Clarendon Press

Frolov, A. O., Mylnikov, A. P. \& Malysheva, M. N. (1997). Description and electron microscopical study of the free-living cryptobiid flagellate, Dimastigella mimosa sp. n. (Kinetoplastida, Cryptobiidae). Tsitologija 39, 442-453 (in Russian).

Haag, J., O'hUigin, C. \& Overath, P. (1998). The molecular phylogeny of trypanosomes: evidence for an early divergence of the Salivaria, Mol Biochem Parasitol 91, 37-49.

Hollar, L. \& Maslov, D. A. (1997). A phylogenetic view on the genus Phytomonas, Mol Biochem Parasitol 89, 295-299.

Hollar, L., Lukeš, J. \& Maslov, D. A. (1998). Monophyly of endosymbiont containing trypanosomatids: phylogeny versus taxonomy, J Eukaryot Microbiol 45, 293-297.

Kishino, H. \& Hasegawa, M. (1989). Evaluation of the maximum likelihood estimate of the evolutionary tree topologies from DNA sequence data, and the branching order in Hominoidea, J Mol Evol 29, 170-179.

Kivic, P. A. \& Walne, P. L. (1984). An evaluation of a possible phylogenetic relationship between the Euglenophyta and Kinetoplastida, Origins Life 13, 269-288.

Landweber, L. F. \& Gilbert, W. (1994). Phylogenetic analysis of RNA editing: a primitive genetic phenomenon, Proc Natl Acad Sci USA 91, 918-921.

Laveran, A. \& Mesnil, F. (1901). Sur les flagellés à membrane ondulante des poissons (genres Trypanosoma Gruby et Trypanoplasma n.gen.), C R Acad Sci Paris 133, 670-675.

Léger, L. (1904). Sur les affinités de l'Herpetomonas subulata et la phylogénie des trypanosomes, C R Sean Soc Biol Ses Fil 56, 615-617.

Lom, J. (1979). Biology of the trypanosomes and trypanoplasms of fish. In Biology of the Kinetoplastida, vol. 2, pp. 269-337. Edited by W. H. R. Lumsden \& D. A. Evans. London: Academic Press.

Lukeš, J., Arts, G. J., van den Burg, J., de Haan, A., Opperdoes, F., Sloof, P. \& Benne, R. (1994). Novel pattern of editing regions in mitochondrial transcripts of the cryptobiid Trypanoplasma borreli, EMBO J 13, 5086-5098.

Lukeš, J., Jirků, M., Doležel, D., Kraĺová, I., Hollar, L. \& Maslov, D. A. (1997). Analysis of ribosomal RNA genes suggests that trypanosomes are monophyletic, $J$ Mol Evol 44, 521-527.
Lukeš, J., Jirků, M., Avliyakulov, N. \& Benada, O. (1998). Pankinetoplast DNA structure in a primitive bodonid flagellate, Cryptobia helicis, EMBO J 17, 838-846.

Maslov, D. A. \& Simpson, L. (1994). RNA editing and genomic organization in the cryptobiid kinetoplastid protozoan, Trypanoplasma borreli, Mol Cell Biol 14, 8174-8182.

Maslov, D. A., Avila, H. A., Lake, J. A. \& Simpson, L. (1994). Evolution of RNA editing in kinetoplastid protozoa, Nature 365, 345-348.

Maslov, D. A., Lukeš, J., Jirků, M. \& Simpson, L. (1996). Phylogeny of trypanosomes as inferred from the small and large subunit rRNAs: implications for the evolution of parasitism in the trypanosomatid protozoa, Mol Biochem Parasitol 75, 197-205.

Maslov, D. A., Yasuhira, S. \& Simpson, L. (1999). Phylogenetic affinities of Diplonema within the Euglenozoa as inferred from the SSU rRNA gene and partial COI protein sequences, Protist 150, 33-42.

Minchin, E. A. (1908). Investigation on the development of trypanosomes in tse-tse flies and other Diptera, $Q J$ Microsc Sci 52, 159-260.

Mylnikov, A. P. (1986). The ultrastructure of the flagellate Parabodo nitrophilus (Bodonina). Tsitologija 28 1056-1062 (in Russian)

Nerad, T. A. (1993). ATCC Catalogue of Protists, 18th edn. Rockville: America Type Culture Collection Press.

Olsen, G. J. (1990). Sequence editor and analysis program. Computer program distributed by the author. University of Illinois, Urbana, IL, USA.

Philippe, H. \& Adoutte, A. (1998). The molecular phylogeny of Eukaryota: solid facts and uncertainties. In Evolutionary Relationships Among Protozoa, pp. 25-56. Edited by G. H. Coombs, K. Vickerman, M. A. Sleigh \& A. Warren. Dordrecht: Kluwer Academic Publishers.

Simpson, A. G. B. (1997). The identity and composition of the Euglenozoa, Arch Protistenkd 148, 318-328.

Sogin, M. L., Elwood, H. \& Gunderson, J. (1986). Evolutionary diversity of eukaryotic small-subunit rRNA genes, Proc Natl Acad Sci U S A 83, 1383-1387.

Stevens, J. R., Noyes, H. A., Dover, G. A. \& Gibson, W. C. (1999). The ancient and divergent origins of the human pathogenic trypanosomes, Trypanosoma brucei and T. cruzi, Parasitology 118, 107-116.

Swofford, D. L. (1998). PAUP* 4.0: phylogenetic analysis using parsimony (and other methods), beta version. Sunderland, MA: Sinauer Associates.

Swofford, D. L., Olsen, G. J., Waddell, P. J. \& Hillis, D. M. (1996). Phylogenetic inference. In Molecular Systematics, pp. 407-514. Edited by D. M. Hillis, C. Moritz \& B. K. Mable. Sunderland, MA: Sinauer Associates.

Vickerman, K. (1976). The diversity of the Kinetoplastid flagellates. In Biology of the Kinetoplastida, pp. 1-34. Edited by W. H. R. Lumsden \& D. A. Evans. London: Academic Press. Vickerman, K. (1978). The free-living trypanoplasms: description of three species of the genus Procryptobia n. g., and redescription of Dimastigella trypaniformis Sandon, with notes on their relevance to the microscopic diagnosis of disease in man and animal, Trans Am Microsc Soc 97, 485-502.

Vickerman, K. (1994). The evolutionary expansion of the trypanosomatid flagellates, Int J Parasitol 24, 1317-1331.

Wiemer, E. A. C., Hannaert, V., van den ljssel, P. R. L. A., van Roy, 
J., Opperdoes, F. R. \& Michels, P. A. M. (1995). Molecular analysis of glyceraldehyde-3-phosphate dehydrogenase in Trypanoplasma borreli: an evolutionary scenario of subcellular compartmentation in Kinetoplastida, J Mol Evol 40, 443-454.

Woo, P. T. K. (1994). Flagellate parasites of fishes. In Parasitic Protozoa, pp. 1-80. Edited by J. T. Kreier. London: Academic Press.

Wright, A.-D. G., Li, S., Feng, S., Martin, D. S. \& Lynn, D. H. (1999). Phylogenetic position of the kinetoplastids, Cryptobia bullocki,
Cryptobia catostomi, and Cryptobia salmositica and monophyly of the genus Trypanosoma inferred from small subunit ribosomal RNA sequences, Mol Biochem Parasitol 99, 69-76.

Yasuhira, S. \& Simpson, L. (1996). Guide RNAs and guide RNA genes in the cryptobiid kinetoplastid protozoan, Trypanoplasma borreli, RNA 2, 1153-1160.

Zhukov, B. F. (1975). A new flagellate Bodo sorokini sp. n. (Bodonina, Kinetoplastida). Biol Inland Waters Bull 25, 23-25 (in Russian). 